\title{
Study on a Dynamic Intelligent Control Algorithm of Data Transfer for Moving Object
}

\author{
Fenqin $\mathrm{He}^{1,2}$ Jianzhong Yin ${ }^{3,4}$ \\ ${ }^{1}$ Institute of Land Reclamation and Ecological Restoration, China University of Mining and Technology, Beijing \\ 100083, P. R. China \\ ${ }^{2}$ School of Environmental Science and Safety Engineering, Tianjin University of Technology, Tianjin 300191, P. R. \\ China \\ ${ }^{3}$ Transportation Research Center(TRC), Wuhan University, Wuhan 430079, P. R. China \\ ${ }^{4}$ State Key Laboratory of Information Engineering in Surveying, Mapping and Remote Sensing (LIESMRS), Wuhan \\ University, Wuhan 430079, P. R. China
}

\begin{abstract}
Starting with data transfer, before transmitting GPS data of moving destination terminal to the monitoring center, dynamic intelligent algorithm can control the rhythm of data transfer, filter the unnecessary data transfer, and recover the filtered data. It puts forward the control model and algorithm of data transfer for moving destination terminal, and discusses the condition of judgment and method of data restoration involved with the algorithm. The testing result shows that effect of the presented algorithm is evident, and data transmission rate is ideal. Furthermore, the recovered data can magnificently approach the original GPS trajectories.
\end{abstract}

Keywords: Moving object, Data transfer, Dynamic intelligent control, Algorithm

\section{Introduction}

With the rapid economic development and market prosperity, traffic congestion has become a serious problem due to unprecedented busy transportation and all kinds of vehicles increasing. Integrating spatial information technology with communication technology, intelligent transportation system(ITS) not only greatly improves the traffic problem, but also can ameliorate global environment and promote the economy sustainable development [1]. Navigation and monitoring integration system(NMIS) is a typical application of integrating spatial information technology with communication technology. The vehicle terminal of the NMIS is a sort of moving object. In recent years, with the development of global position system(GPS), vehicle real-time locating has become a reality. Wireless correspondence technology has a smashing evolution and General Packet Radio
Service(GPRS) has a characteristic of on-line for ever, whenever or wherever, people can easily access to internet. With the unceasing development of wireless access technique, the bottle-neck of mobile spatial information service has been eliminated, remote monitoring and dispatching vehicle has become a realization [2].

When the vehicle terminal of NMIS navigates, it transmits information with the monitoring center each other at the same time. That is to say, when the vehicle terminal sends position and status information to the monitoring center, it receives the dictate and real-time traffic information from the monitoring center synchronously.

NMIS is a sort of high-tech system, which integrates GPS, geographical information system(GIS) and mobile communication technique. Its main function includes as follows: (1)sending dynamic location(longitude, latitude), time and status information of moving object equipped GPS receiver to the monitoring center by wireless communication link; (2)displaying the trajectory of moving object on the digital map, which has the powerful geographical information query function; (3)monitoring the objective information interested by the user, such as location, speed, moving direction, vehicle status, and so on; (4)providing visual effect for vehicle scheduling management and vehicle guidance in order to improve the vehicle operating efficiency and ensure the security of vehicle.

Since last century 90's early times, vehicle monitoring system equipped GPS has begun to develop in China. Until 1999 year, its market has occurred to rapid incremental tendency, and become maturation step by step. Furthermore, its technology makes a mighty advance and development in China at the same time [3]. While, compared with developed country, research and development of vehicle navigation and monitoring begins relatively late in China, thus it is 
underway phase at present, which results in the delay of navigation and monitoring production, whether the development of technology or application system in China [4].

\section{Problem}

In NMIS, vehicle terminal sends information of vehicle location and status to the monitoring center, and then the monitoring center sends scheduling, dictate or guidance information to separate vehicle terminal. Thus, data transfer between vehicle terminal and the monitoring center plays an important role in the whole system. Considering that vehicle terminal is moving, it has to adopt wireless mode to exchange data with the monitoring center. At present, domestic and overseas applications almost adopt GPRS, whose transfer speed is $171.2 \mathrm{Kbps}$ in theory, while almost city provides transfer speed with $40 \sim 60 \mathrm{Kbps}$ in practice [5]. The wireless communication of NMIS has a characteristic itself, namely, time needs very short in a call, and yet call frequency is high.

The characteristic of the data sent to the monitoring center by NMIS is that the data quantity is large and the data type is various, simultaneously, also has the certain request of time limit. The real-time data information dynamically renews. Moreover, the data is progressive with the lapse of time. The quantity of historical data is huge, and the retention time is long [6]. Obviously, in NMIS, if the large-scale goal is concurrent, it can give the communications system to serious burden for data transfer, and also influences real-time and reliability. Furthermore, the huge historical data brings the serious burden to memory system of the monitoring center.

In fact, NMIS is such a system, which can carry out storage management, query and analysis, scheduling and guidance to the moving object for changing spatial location with time efflux. Between monitoring data and traditional database data has an obvious difference, namely, the value of vehicle position is continuous and dynamic change, which directly results in a massive data and the lower efficiency of system query [7]-[10]. When the moving object transmits data to the monitoring center, the selected strategy of time interval occupies more unnecessary communication bandwidth, and the model is too simple for the space time [11]-[13]. Wang Shengsheng has studied on the moving object model based on vehicle monitoring system, and put forward a sort of improved model for moving object, which provided a dynamic time interval selecting algorithm. This algorithm can dynamically adjust time density based on the moving speed of the object, and effectively reduce the communication times [14].
Wang Weijing has studied on design and implementation of spatial-temporal data model in vehicle monitoring system. Aiming at the characteristic of high changing frequency of vehicle object trajectory data and massive data in the vehicle monitoring system, on the basis of spatial-temporal cube model, he puts forward spatial-temporal cube sliced, cell of spatialtemporal cube built, and modeling idea of trajectory version, which improves the capability of storage and spatial-temporal query [15].

In brief, starting with data transfer, before transmitting GPS data of moving destination terminal to the monitoring center, dynamic intelligent algorithm can control the rhythm of data transfer, also filter the unnecessary data transfer, and recover the filtered data.

\section{Algorithm}

To reduce the unnecessary data transfer and bandwidth occupation, the dynamic intelligent control method judges whether the data of vehicle terminal needs to be sent to the monitoring center or not, this article designs the dynamic intelligent control algorithm of vehicle terminal data transfer.

\subsection{Algorithm design}

Starting with the GPS real-time raw data, this paper gains road section ID information of the GPS points and designs the algorithm by using map matching technique of the navigation function and the navigation digital map resources. Whether the GPS points have to be sent to the monitoring center is controlled by three judge condition, namely, road section ID of the GPS point subordinate, changing quantity of GPS point speed and GPS point direction. The main thought is that the first point of each road section is necessary sent to the monitoring center, then the points, whose changing quantity of speed and direction exceeds the assigned threshold value, have to be sent to the monitoring center, otherwise, given up. The order of condition judgment shows as follows. Firstly, if the road section of current point is a new one, the point has to be sent to the monitoring center, otherwise, controlled by the second judgment condition. Secondly, comparing with the last point sent to the monitoring center, if changing quantity of current point speed surpasses the assigned threshold value, the point has to be sent to the monitoring center, otherwise, controlled by the third judgment condition. Thirdly, comparing with the last point sent to the monitoring center, if changing quantity of current point direction surpasses the assigned threshold value, the point has to be sent to the monitoring center, otherwise, the flow enters the 
next collected GPS point. The flow design of data transfer control algorithm for vehicle terminal is shown as Fig.1.

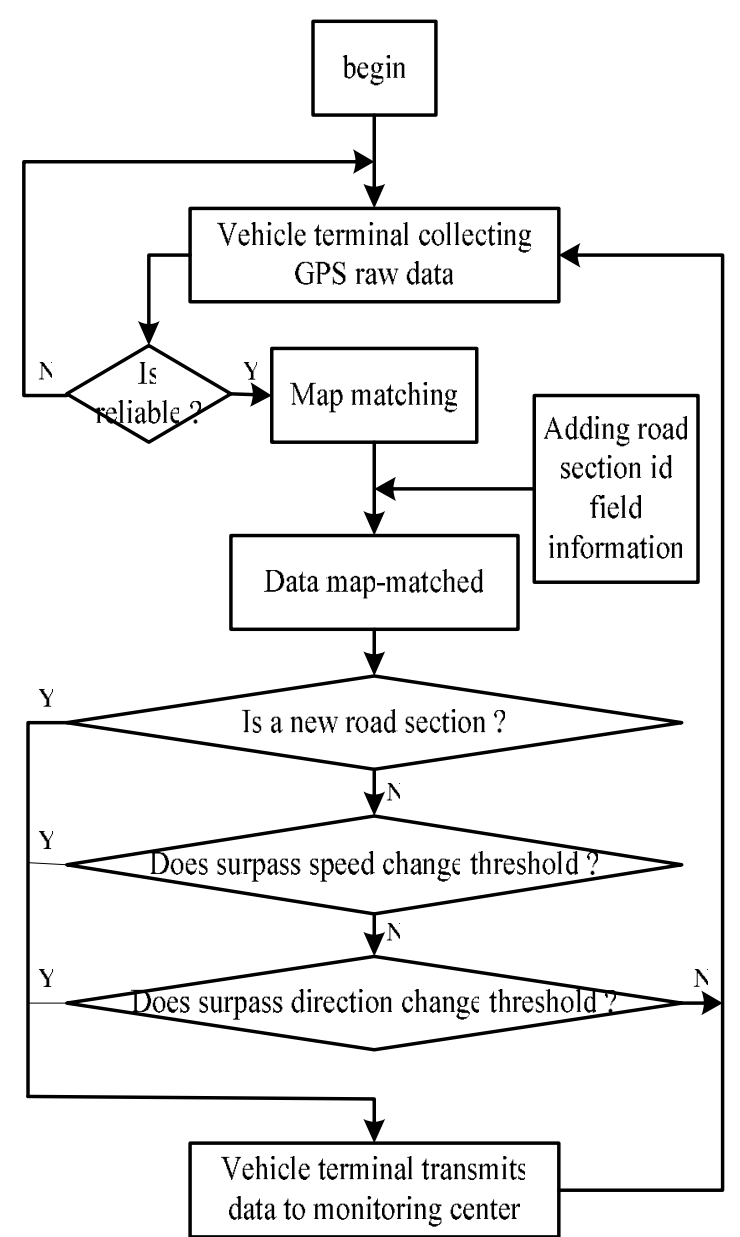

Fig. 1: The design flowchart of dynamic intelligent control algorithm of data transfer for moving object

\subsection{Algorithm description}

The step description of dynamic intelligent control algorithm of data transfer for moving object is indicated as follows.

The step 1, the vehicle terminal GPS receiver obtains current locating point information.

The step 2, examining the number of satellite whether it is more than 4 for the current locating point, if it conforms to the condition, we may think that the data is effective, the algorithm flow enters the step 3, otherwise, returns the step 1.

The step 3, the vehicle terminal carries on the map matching to the current locating point with the navigation digital map.
The step 4, adding road section ID information to the current locating point matched, algorithm flow gains the matched data.

The step 5, judging the locating point whether it enters the new road section, if condition is true, algorithm flow enters the step 8 , otherwise, enters the step 6.

The step 6, comparing with the last point sent to the monitoring center, if changing quantity of current point speed surpasses the assigned threshold value, algorithm flow enters the step 8 , otherwise, enters the step 7.

The step 7, comparing with the last point sent to the monitoring center, if changing quantity of current point direction surpasses the assigned threshold value, algorithm flow enters the step 8, otherwise, enters the step 1.

The step 8, sending the current point to the monitoring center, algorithm flow enters the step 1 in order to obtain the next GPS raw data.

\section{Judgment condition and data recovery}

The threshold value of changing quantity of speed and direction is confirmed by experience. While the changing quantity of speed, the changing quantity of direction and the threshold value need satisfy the following relations. The starting point is to guarantee that the filtered data between the two transmission points can be recovered by linear interpolation method in a certain error range.

\subsection{Changing quantity of speed}

The judgment condition of changing quantity of speed should guarantee that the data between two neighboring transmission points can be recovered by linear interpolation method in a certain error range. The threshold value of error is confirmed by experience. Judgment equation is expressed as formula (1).

$$
\frac{\sum_{i=1}^{n}\left|v_{0}+\frac{v_{n}-v_{0}}{n} \cdot i-v_{i}\right|}{n}<\varepsilon_{v}
$$

Where $v_{0}$ is the speed true value of previous transmission GPS point. $v_{n}$ is the speed true value of current GPS point. $v_{i}$ is the speed true value of the $i$ th GPS point after the previous transmission point. $n$ is the sequence number of the current transmission point, which is counted from the first effective GPS point after the previous transmission point. $i$ is the sequence number of the effective GPS point. $\boldsymbol{\varepsilon}_{v}$ is the permission threshold value of error. 


\subsection{Changing quantity of direction}

The judgment condition of changing quantity of direction should guarantee that the data between two neighboring transmission points can be recovered by linear interpolation method in a certain error range. The threshold value of error is confirmed by experience. Judgment equation is shown as formula (2).

$$
\frac{\sum_{i=1}^{n}\left|d i r_{0}+\frac{d i r_{n}-d i r_{0}}{n} \cdot i-d i r_{i}\right|}{n}<\theta_{d i r}
$$

Where $d i r_{0}$ is the direction true value of previous transmission GPS point. $d i r_{n}$ is the direction true value of current GPS point. dir $r_{i}$ is the direction true value of the $i$ th GPS point after the previous transmission point. $n$ is the sequence number of the current transmission point, it is counted from the first effective GPS point after the previous transmission point. $i$ is the sequence number of the effective GPS point. $\theta_{d i r}$ is the permission threshold value of error.

\subsection{Data recovery}

According to the control algorithm of data transfer, the data is processed, and then is sent to the monitoring center. However, the data is already not the most primitive continual data GPS gathered. If the trajectory is redisplayed or the position of the vehicle is queried in some time, the filtered data has to be recovered by the received data in the monitoring center.

The restoration of speed data uses the linear interpolation means, whose equation is written as formula (3).

$$
v_{i}^{\prime}=v_{0}+\frac{v_{n}-v_{0}}{n} \cdot i
$$

Where $v_{i}^{\prime}$ is the restoration value of speed for the $i$ th GPS point after previous transmission GPS point. The significance of other variables is the same as formula (1).

The restoration of direction data uses the linear interpolation means, whose equation is expressed as formula (4).

$$
d i r_{i}^{\prime}=d i r_{0}+\frac{d i r_{n}-d i r_{0}}{n} \cdot i
$$

Where $d i r_{i}^{\prime}$ is the restoration value of direction for the $i$ th GPS point after previous transmission GPS point. The significance of other variables is the same as formula (2).

The space coordinate of the GPS point between two neighboring transmission points can be recovered by the following equation, which is defined as formula (5). $\left\{\begin{array}{l}x_{i}^{\prime}=x_{0}+p_{i} \cdot \cos d i r_{i}^{\prime} \\ y_{i}^{\prime}=y_{0}+p_{i} \cdot \sin d i r_{i}^{\prime}\end{array}\right.$
and here $p_{i}=\frac{\sqrt{\left(y_{n}-y_{0}\right)^{2}+\left(x_{n}-x_{0}\right)^{2}}}{n} \cdot i$

Where $x_{i}$ is the $x$ coordinate restoration value of the $i$ th GPS point after the previous transmission point. $y_{i}{ }^{\prime}$ is the $y$ coordinate restoration value of the $i$ th GPS point after the previous transmission point. $x_{0}$ is the $x$ coordinate restoration value of the previous GPS transmission point. $y_{0}$ is the $y$ coordinate restoration value of the previous GPS transmission point. $x_{n}$ is the $x$ coordinate true value of current GPS point. $y_{n}$ is the $y$ coordinate true value of current GPS point. $d i r^{\prime}{ }^{\prime}$ is the direction restoration value of the $i$ th GPS point after previous transmission GPS point. $n$ is the sequence number of the current transmission point, which is counted from the first effective GPS point after the previous transmission point. $i$ is the sequence number of the effective GPS point.

\section{Experimental result}

In the $\varepsilon_{v}$ and $\theta_{d i r}$ preconcerted error range, according to the dynamic intelligent control algorithm of data transfer for moving object, the paper does the experimentation with 6,208 points of GPS raw realtime data. The test result shows that the original GPS trajectories of 6,028 points only transmit 218 points, the data transmission rate is only $3.6 \%$, and the recovered data can approach the original GPS trajectories in the monitoring center. The experiment proves that the algorithm proposed in this paper can well control the vehicle terminal data before it is sent to the monitoring center.

\section{Conclusions}

The article expounds the design and description of the dynamic intelligent control algorithm of data transfer for moving object, also proposes the method for confirming the judgment conditions of the speed changing quantity and the direction changing quantity involved into the algorithm, and puts forward the equation for recovering data in the monitoring center. Finally, the experimental result shows that the algorithm has an excellent effect on data transmission rate, that is to say, the dynamic intelligent control algorithm of data transfer for moving object reduces the data transmission rate to only $3.6 \%$. In addition, the recovered data can approach the original GPS trajectories in the monitoring center. This method greatly reduces the bandwidth occupancy of data 
transfer, and alleviates stress for storage space for historical data.

\section{Acknowledgement}

This work is partially supported by the New-Century Outstanding Young Scientist Program of china (Grant No. NCET-04-0484).

\section{References}

[1] D.R. Li and Q.Q. Li, The technique integration of the spatial information and communication. Geomatics and Information Science of Wuhan University, 26:1-7, 2001.

[2] D.R. Li, Q.Q. Li, Z.Y. Xie and X.Y. Zhu, The technique integration of the spatial information and mobile communication. Geomatics and Information Science of Wuhan University, 27:1-8, 2002.

[3] B. Yang, editor. Intelligent transportation system, China Railway Publishing House, Beijing, 2000.

[4] Q.Q. Li, B.J. Li and J.H. Li, Vehicle navigation technology today and trends. Engineering of Surveying and Mapping, 14:4-7, 2005.

[5] Y. Ursunjan, Q. Chang, S.L. Mei and Y.D. Zhou, Design and implementation of an intelligent vehicle monitoring system based on GPRS. Video Engineering, 5:114-117, 2005.

[6] B.S. Yang and Q.Q. Li, An algorithm for progressive transmission vector map data over the WWW. Acta Geodaetica et Cartographica Sinica, 34:355-359, 2005.

[7] H.D. Chon, D. Agrawal and A.E. Abbadi, Storage and retrieval of moving objects. Proceedings of the International Conference on Mobile Data Management, pp.173-184, 2001.

[8] L. Forlizzi and R.H. Guting, A data model and data structures for moving objects databases. Proceedings of ACM SIGMOD International Conference on Management of Data, pp.99-101, 2000.

[9] R.H. Guting and M.H. Bohlen, A foundation for representing and querying moving objects. $A C M$ Transactions on Database Systems, 25:70-75, 2000.

[10] O. Wolfson, B. Xu and S. Chamberlain, Moving object database: issue and solutions. Proceedings of 10th International Conference on Science and Statistical Database Management, pp.111-122, 1998.

[11] H.D. Chon, D. Agrawal and A.E. Abbadi, FATES: finding a time dependent shortest path. Proceedings of the 4th International Conference on MDM, pp.165-180, 2003.

[12] Y. Chen, F.Y. Rao, X.L. Yu and D. Liu, CAMEL: a moving object database approach for intelligent location aware services. Proceedings of the 4th International Conference on MDM, pp.331-334, 2003.

[13] M. Vazirgiannis and O. Wolfson, A spatiotemporal model and language for moving objects on road networks. Symposium of Advances in Spatial and Temporal Databases, pp.20-35, 2001.

[14] S.S. Wang and D.Y. Liu, Moving object model based vehicle monitor system. Chinese Journal of Scientific Instrument, 25:509-511, 2004.

[15] W.J. Wang, J.N. Weng and K. Fan, Design and implementation of spatial-temporal data model in vehicle monitor system. Computer Engineering and Design, 27:1042-1045, 2006. 